\title{
Combination of platelet-rich plasma and bone marrow mesenchymal stem cells enhances tendon-bone healing in a rabbit model of anterior cruciate ligament reconstruction
}

\author{
Chong Teng ${ }^{1}$, Chenhe Zhou ${ }^{2}$, Danfeng $\mathrm{Xu}^{2}$ and Fanggang Bi ${ }^{3^{*}}$
}

\begin{abstract}
Background: The objective of this study was to investigate the potency of platelet-rich plasma (PRP) combined with bone marrow mesenchymal stem cells (BMSCs) to promote tendon-bone healing in a rabbit model.

Methods: In the in vitro study, the effects of PRP on osteogenic induction of BMSCs were analysed. Later, PRP with or without BMSCs was used in the rabbit model of anterior cruciate ligament reconstruction. Specimens were harvested 8 weeks postoperatively to evaluate tendon-bone healing by histology, radiology, and biomechanical testing.

Results: The in vitro study revealed that collagen I, osteocalcin, and osteopontin expression was higher in BMSCS co-cultured with PRP for 14 days. The in vivo study revealed a more mature tendon-bone interface using light microscopy, a more newly formed bone at the bone tunnel walls detected by micro-computed tomography, and a significantly higher failure load as assessed by biomechanical testing in the BMSC + PRP group than in the control and PRP groups.
\end{abstract}

Conclusions: These results indicate that the combination of PRP and BMSCs promotes tendon-bone healing and has potential for clinical use.

Keywords: Platelet-rich plasma, Bone marrow mesenchymal stem cells, Anterior cruciate ligament, Reconstruction, Tendon-bone healing

Abbreviations: $\mathrm{ACL}$, Anterior cruciate ligament; BMSCs, Bone marrow mesenchymal stem cells; PRP, Platelet-rich plasma; B-PT-B, Bone-patellar tendon-bone; PDGF, Platelet-derived growth factor; PDEGF, Platelet-derived epidermal growth factor; TGF, Transforming growth factor; VEGF, Vascular endothelial growth factor; DMEM, Dulbecco's modified Eagle medium; EDTA, Ethylenediaminetetraacetic acid; H\&E, Haematoxylin and eosin; Micro-CT, Micro-computed tomography; RT-PCR, Real-time quantitative reverse transcription-polymerase chain reaction; GFP, Green fluorescence protein

\footnotetext{
*Correspondence: 163bfg@163.com

${ }^{3}$ Department of Orthopaedic Surgery, The First Affiliated Hospital of

Zhengzhou University, Zhengzhou 450001, China

Full list of author information is available at the end of the article
} 


\section{Background}

Anterior cruciate ligament (ACL) injuries are common, frequently lead to knee disability, and have low intrinsic regenerative ability [1]. ACL reconstruction is the gold standard because of its positive outcomes. Hamstring tendon grafts have been widely used due to the lower donorsite morbidity compared to bone-patellar tendon-bone (B-PT-B) grafts [2]. However, tendon-bone healing between a hamstring tendon graft and the created bone tunnels is associated with significantly slower bone healing compared with the B-PT-B graft [3]. Therefore, means for improving tendon-bone healing after reconstruction have become a major research focus in sports medicine [4].

Platelet-rich plasma (PRP), an autologous enriched source of various growth factors, such as platelet-derived growth factor (PDGF), platelet-derived epidermal growth factor (PDEGF), platelet-derived angiogenesis factor, transforming growth factor (TGF), insulin-like growth factor, and vascular endothelial growth factor (VEGF), has been widely used during trauma and orthopaedic surgery $[5,6]$. PRP can clinically accelerate the healing of hard and soft tissues after maxillofacial, plastic, dermatologic, dental, and orthopaedic surgeries [7]. However, existing preclinical and clinical evidence regarding PRP use in ACL surgery failed to demonstrate a clear benefit [8]. Bone marrow mesenchymal stem cells (BMSCs) have attracted much interest and have become ideal seed cells for tissue engineering because of their multipotentiality and self-renewal potential, as well as their possible suitability for clinical use [9]. BMSCs have outstanding potential to promote tendon regeneration [10], and their osteogenic differentiation potential in vitro and bone formation capability on biodegradable scaffolds in vivo have been characterised in several studies $[11,12]$. Many studies have shown that host cells containing BMSCs from surrounding bone tunnel bone marrow contribute to tendon-bone healing $[13,14]$. However, BMSCs in bone marrow are rare, with only one BMSC detected in $1 \times 10^{5}$ bone marrow mononuclear cells. PRP, as a storage vehicle for growth factors such as PDGF, TGF- $\beta$, PDEGF, VEGF, and platelet factor-4, enhances osteogenic differentiation of BMSCs [15]. Many studies have shown that factors that positively affect bone formation and fracture healing also positively affect tendon-bone healing [16].

In the present study, we seeded PRP and BMSCs at the tendon-bone interface using fibrin glue to explore whether tendon-bone healing is promoted by providing BMSCs and an osteoinductive factor.

\section{Methods}

\section{Preparation of PRP}

Autologous PRP was prepared as described previously [17]. Briefly, $10 \mathrm{~mL}$ of whole blood was drawn from the marginal auricular vein using an 18-gauge catheter. The blood was injected into a sterile centrifuge tube containing $1.5 \mathrm{~mL}$ of sodium citrate. The mixture was centrifuged at $1200 \times g$ for $10 \mathrm{~min}$ to separate the plasma from the red blood cells. The plasma was centrifuged again at $2500 \times g$ at $4{ }^{\circ} \mathrm{C}$ for $20 \mathrm{~min}$, and the precipitated platelets (1 mL) were collected.

\section{Isolation and culture of BMSCs}

BMSCs were generated from bone marrow aspirates of New Zealand White rabbits (age, 12 weeks; weight, $2.5 \pm 0.2 \mathrm{~kg})$, as described previously [18]. Mononuclear cells were collected after centrifugation in Ficoll-Hypaque gradient (Sigma Co., St. Louis, MO, USA) and resuspended in Dulbecco's modified Eagle medium (DMEM) containing $10 \%$ fetal bovine serum (FBS; Gibco, Grand Island, NY, USA). After a 72-h incubation at $37{ }^{\circ} \mathrm{C}$ in $5 \% \mathrm{CO}_{2}$, the non-adherent cells were removed by changing the culture medium. Adherent cells were subcultured when they reached 70-80 \% confluence. A homogenous BMSC population was obtained after 2 weeks of culture, and the third passage was harvested for further use. The passage 3 cells were identified by detecting surface antigen marker expression profiles using flow cytometry. The osteogenic, chondrogenic, and adipogenic differentiation abilities of the cells were determined using inducing media for 3 weeks. Alizarin red, oil red $\mathrm{O}$, and alcian blue staining were performed.

\section{Induction of osteogenic gene expression by PRP}

Third passage BMSCs were harvested by trypsinisation and centrifugation. After culturing for $24 \mathrm{~h}$, the original culture medium was removed. The BMSCs were washed three times with PBS and incubated in DMEM with $10 \%$ PRP. In the control group, BMSCs were incubated in DMEM with $10 \%$ FBS. Total RNA was extracted from cells cultured for 3, 7, and 14 days using TRIzol reagent (Invitrogen, Carlsbad, CA, USA). RNA concentration was determined with the NanoDrop spectrophotometer (NanoDrop Technologies, Wilmington, DE, USA), and $200 \mathrm{ng}$ of RNA was used to synthesise complementary DNA (cDNA) using the iScript cDNA synthesis kit (Bio-Rad Laboratories, Hercules, CA, USA). The Stratagene M×3000P system (Stratagene, La Jolla, CA, USA) was used to perform and monitor the reactions. The QuantiTect SYBR Green PCR kit (Qiagen, Valencia, CA, USA) was used to quantify transcription levels of osteogenic genes, including collagen I, osteocalcin, and osteopontin. The glyceraldehyde-3-phosphate dehydrogenase gene was amplified in parallel with the target genes. The primer sequences are listed in Table 1. 
Table 1 Primers of collagen I, OCN, and OPN used in RT-PCR in this study

\begin{tabular}{lll}
\hline Gene & & Primer sequence \\
\hline GAPDH & Forward & 5'-ATGGGGAAGGTGAAGGTCG-3' \\
& Reverse & 5'-TAAAAGCAGCCCTGGTGACC-3' \\
Collagen I & Forward & 5'-GGTTGTTGAAGAGGCTG-3' \\
& Reverse & 5'-GATGGCCTGAAGCTCAA-3' \\
Osteocalcin & Forward & 5'-CCGGGAGGAGATCTGTGAAA-3' \\
& Reverse & 5'-CTGCCTTCTTCCACAATTTATCC-3' \\
Osteopontin & Forward & 5'-GCCAGTTGCAGCCTTCTCA-3' \\
& Reverse & 5'-GCCATGCCCAAGAGACAAAA-3' \\
\hline
\end{tabular}

\section{$\mathrm{ACL}$ reconstruction model in rabbits}

Animal experiments were approved by the Zhejiang University Ethics Committee. A total of 30 New Zealand White rabbits (age, 12 weeks, and weight, $2.5 \pm 0.2 \mathrm{~kg}$ ) were used in this study. All rabbits underwent ACL reconstruction in the left hind leg after intravenous anaesthesia with $30 \mathrm{mg} / \mathrm{kg}$ body weight pentobarbital sodium solution (Dawen Biotech, Seoul, Korea). After shaving and disinfecting the left hind leg, a lateral parapatellar incision was made to expose the knee joint. After the native ACL was excised, the tibial and femoral bone tunnels were created with a 2.5 -mm-diameter drill (Fig. 1b). The ipsilateral semitendinosus tendon was harvested to reconstruct the ACL (Fig. 1a). The rabbits were divided randomly into three groups. In the first group, a normal hamstring tendon was used for ACL reconstruction (control group). Rabbits from the second group (PRP group) received hamstring tendons wrapped with $0.1 \mathrm{~mL}$ PRP immobilised in $0.1 \mathrm{~mL}$ fibrin glue (TISSEEL kit; Baxter AG, Vienna, Austria) for ACL reconstruction. In the third group (BMSC + PRP group), $1 \times 10^{7}$ BMSCs were immobilised in $0.2 \mathrm{~mL} \mathrm{PRP}$ and $0.1 \mathrm{~mL}$ fibrin glue and used for ACL reconstruction. The PRP concentration and number of BMSCs were determined by flow cytometry. Grafts for the PRP and BMSC + PRP groups were wrapped with glue immediately before insertion into the bone tunnels. Both ends of the graft were fixed by sutures tied over screws in the femur and tibia (Fig. 1c). Animals were allowed to move freely postoperatively. All rabbits were sacrificed with a lethal injection of pentobarbital 8 weeks postoperatively for assessment; half of the specimens in each group ( $n=5$ /group) were used for the histological assessment and the other half ( $n=5$ /group) were used for radiological and biomechanical assessments.

\section{Histology}

The specimens were fixed in $4 \%$ paraformaldehyde for $72 \mathrm{~h}$ after harvest. All samples were decalcified in $10 \%$ EDTA with PBS at room temperature for 4 weeks. The samples were dehydrated through a graded ethanol series, embedded in paraffin wax, and sectioned at $3 \mu \mathrm{m}$ parallel to the longitudinal axis of the bone tunnel. Haematoxylin and eosin (H\&E) and Russell-Movat pentachrome staining were performed to evaluate tendonbone healing for conventional light microscopy.

\section{Radiology and biomechanical testing}

The specimens for radiology and biomechanical testing were frozen at $-80{ }^{\circ} \mathrm{C}$ immediately after harvest. After thawing overnight at $4{ }^{\circ} \mathrm{C}$, the specimens from each group were scanned using a micro-computed tomography $(\mathrm{CT})$ imaging system with a $36-\mu \mathrm{m}$ isotropic voxel resolution under a 60-kV scanning voltage (Skyscan1176; BRUKER, Antwerp, Belgium).
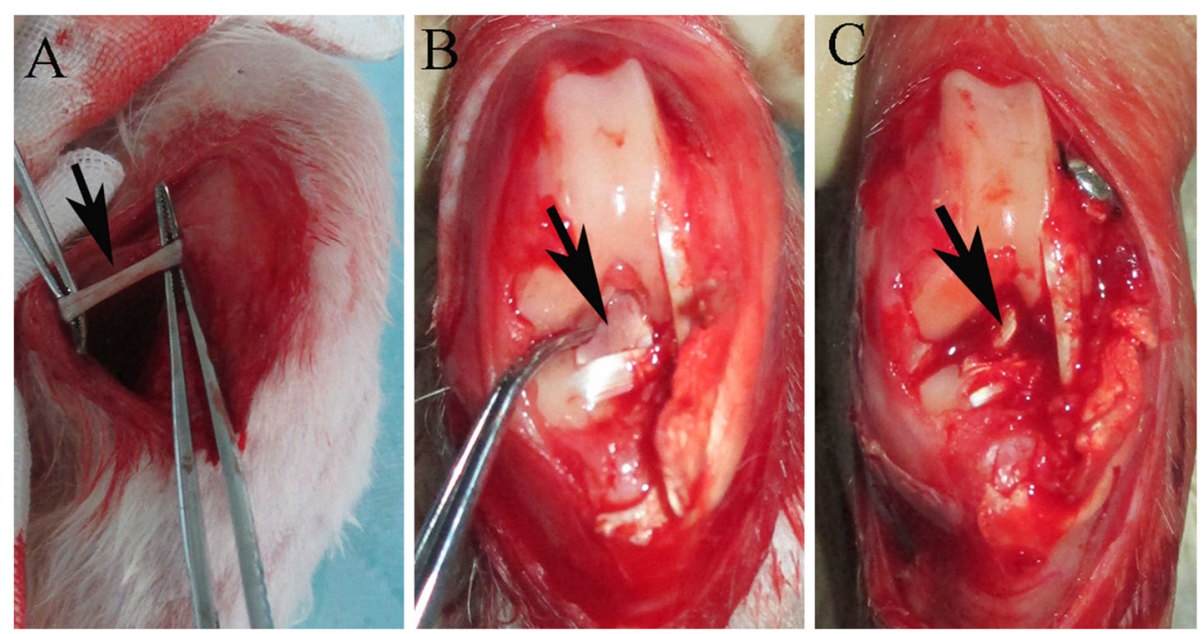

Fig. 1 a Gross observations of the semitendinosus tendon (black arrow) and b native anterior cruciate ligament (ACL) (black arrow). c Macroscopic view of $A C L$ reconstruction (arrow points to autologous semitendinosus tendon graft) 
Biomechanical testing was performed immediately after the scan. All soft tissue except the graft was removed to create a femoral-ACL graft-tibial complex. The femur and tibia were fixed at $45^{\circ}$ flexion in an Instron 553A material testing system (Instron, Norwood, MA, USA; Fig. 2a). The test was performed by increasing the tensile load continuously at a speed of $20 \mathrm{~mm} / \mathrm{min}$. The failure load $(\mathrm{N})$ was recorded by the load-deformation curve, and stiffness $(\mathrm{N} / \mathrm{mm})$ was calculated from the slope of the linear part of the load-deformation curve (Fig. 2b).

\section{Statistical analysis}

All values are expressed as means \pm standard deviation, and the statistical analysis was performed using SPSS software (ver. 16.0; SPSS Inc., Chicago, IL, USA). Differences between groups were detected using one-way analysis of variance followed by Scheffe's multiple comparison test. A $p$ value $<0.05$ was considered significant.

\section{Results}

\section{Identification of BMSCs}

A total of 69.2 and $99.7 \%$ of the passage 3 cultured cells expressed CD44 and CD90, respectively, whereas only $4.82 \%$ expressed CD45 and were identified as BMSCs (Fig. 3). Following 3 weeks of culture in osteogenesis induction medium followed by alizarin red staining, obvious mineralised nodules were observed under the microscope (Fig. 4a). Oil red O staining revealed many lipid droplets (Fig. 4b), and the BMSC cytoplasm was stained green by alcian blue after 3 weeks of chondrogenic induction (Fig. 4c).

\section{Transcription levels of osteogenic genes}

The transcription of osteogenic genes, including collagen I, osteocalcin, and osteopontin, was evaluated by real- time quantitative reverse transcription-polymerase chain reaction (RT-PCR) analysis. Collagen I, osteocalcin, and osteopontin messenger RNA (mRNA) levels in BMSCs increased gradually after co-culture with PRP. The levels of the three osteogenic genes were significantly higher than those in uninduced BMSCs at all time points $(p<$ 0.05, Fig. 5).

\section{Histological observations}

Organised fibrous tissue and some new bone containing chondrocytes were observed at the tendon-bone interface in the control group 8 weeks postoperatively (Fig. 6a, d). Aligned connective tissue, newly formed woven bone, and cartilage were observed at the tendon-bone interface in the PRP group (Fig. 6b, e). A more mature interface with aligned chondrocytes was observed in the BMSC + PRP group, but the fibrous connective tissue at the tendonbone interface was unclear. A more aligned and layered cartilage zone was observed, which incorporated adjacent bone and tendon (Fig. 6c, f).

\section{Micro-CT scan}

The transverse, coronal, and sagittal section images of the tibial bone tunnel were reconstructed with highresolution micro-CT. Newly formed mineralised tissue was evident along the entire length of the bone tunnel by screening slices of each sample. The control microCT images showed no obvious mineralised tissue in the tibial bone tunnels 8 weeks postoperatively (Fig. 7a1-3). Obvious signals were detected in the bone tunnels of the PRP (Fig. 7b1-3) and BMSC + PRP groups (Fig. 7c1-3), indicating mineralised tissue formation at the tendonbone interface. A stronger signal was observed in the BMSC + PRP group than in the PRP group, indicative of more mineralised tissue formation.
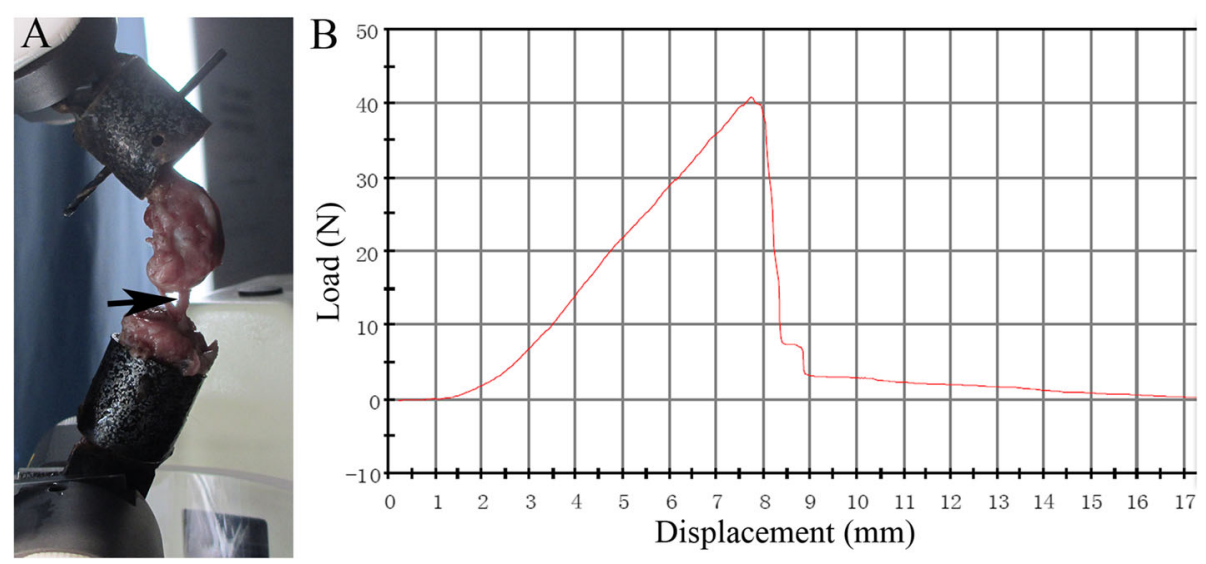

Fig. 2 a The femoral-graft-tibial complex was firmly fixed on the Instron machine to perform the mechanical test (black arrow points to the intra-articular graft). b Representative load-deformation curve obtained by the biomechanical test 

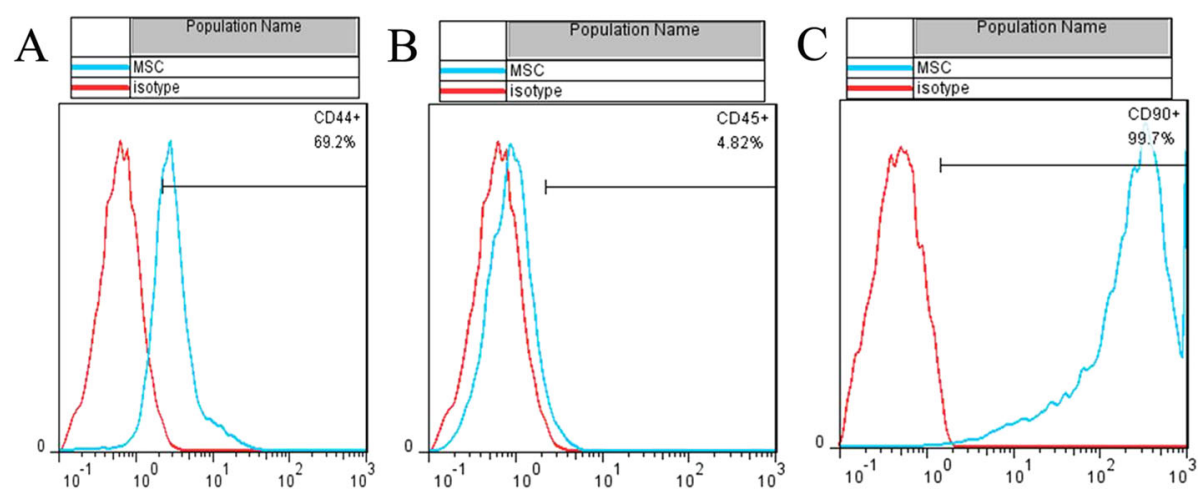

Fig. 3 Flow cytometry analysis of passage 3 cell surface markers: 69.2, 4.82, and $99.7 \%$ positive cells for CD44 (a), CD45 (b), and CD90 (c), respectively

\section{Biomechanical testing}

The failure load (36.22 $\pm 8.77 \mathrm{~N}, n=5)$ was significantly greater in the BMSC + PRP group 8 weeks postoperatively than in the control $(19.56 \pm 2.45 \mathrm{~N}, n=5 ; p=$ $0.001)$ and PRP groups $(24.08 \pm 1.16 \mathrm{~N}, n=5 ; p=$ $0.012)$. No difference was observed between the control and PRP groups ( $p=0.429$; Fig. 8a). No difference in stiffness was observed among the groups (Fig. 8b) (Additional file 1).

\section{Discussion}

This study demonstrated that the combination of PRP and BMSCs enhanced tendon-bone healing in a rabbit model of ACL reconstruction. The aligned and layered cartilage zone revealed that directly inserting the tissue resembled the histology of inserting a native ACL. More mineralised tissue had formed, as detected by micro-CT, and a larger failure load was observed in the BMSC + PRP group, indicative of better osteointegration at the tendon-bone interface. These results indicate that the BMSC + PRP combination is a promising way to promote tendon-bone healing.

BMSCs have the potential for differentiation as multipotent stem cells, but this process is not completely spontaneous. Many studies have shown that some cytokines have stimulatory effects on the differentiation of
BMSCs [19-21]. In the present study, collagen I, osteocalcin, and osteopontin mRNA levels increased gradually when cells were co-cultured with PRP for 14 days. Activated platelets release TGF- $\beta$, PDGF, VEGF, and epidermal growth factor, which promote wound healing [22]. de Mos et al. found that PRP enhanced human tendon cell proliferation and total collagen production in an in vitro study [23].

Tendon-bone healing is slow because the reconstructed tendon graft in the bone tunnel is separated from a vascular supply and the bone is lost at the injury site. During healing, the structure and composition of the native direct tendon-bone interface is not reformed, but a mechanically and structurally inferior interface forms [24]. Previous studies have demonstrated that osteoinductive agents accelerate osteointegration to the tendon graft, improving tendon-bone healing and the mechanical properties [25-27]. Rodeo et al. reported a narrower interface zone in bone morphogenetic proteintreated specimens, indicative of better tendon-bone healing [26]. In the present study, we found that PRP promoted BMSC osteodifferentiation in vitro, and the combination of the two agents promoted tendon-bone healing in vivo.

Successful ACL reconstruction requires solid tendonbone healing. Direct and indirect insertion are the two
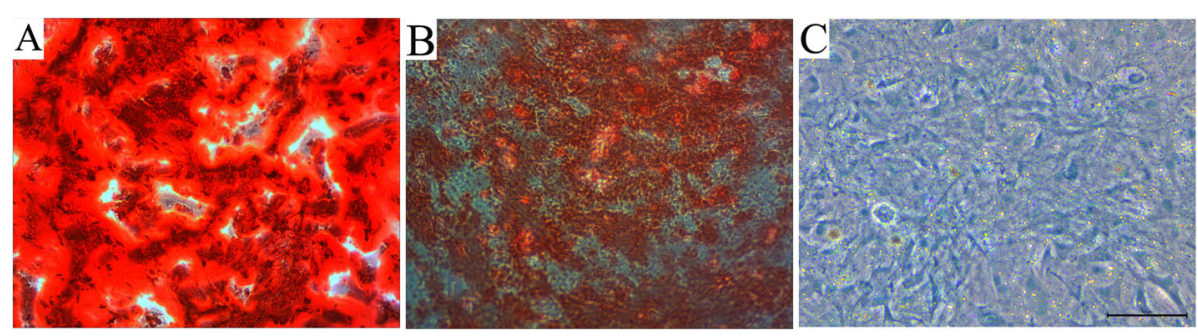

Fig. 4 Alizarin red (a), oil red $\mathrm{O}(\mathbf{b})$, and alcian blue staining (c) to detect the differentiating ability of bone marrow mesenchymal stem cells (BMSCS) relative to the bone, fat, and cartilage, respectively. Scale bar, $50 \mu \mathrm{m}$ 

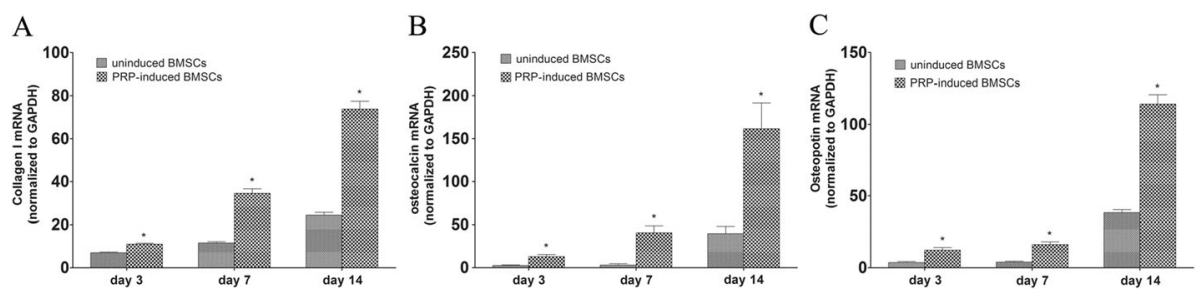

Fig. 5 Reverse transcription-polymerase chain reaction evaluation of osteogenic gene mRNA expression. Asterisk indicates significant difference $(p<0.05)$ compared with gene expression levels of BMSCs co-cultured with or without platelet-rich plasma (PRP) $\mathbf{a}$ collagen I; $\mathbf{b}$ osteocalcin $(\mathrm{OCN})$; c osteopontin (OPN)

types of tendon-bone insertion. Direct insertion, which serves to transmit tensile forces, comprises four typically distinct transition zones, of the bone, mineralised cartilage, fibrocartilage, and ligament. Indirect insertion includes the bone, Sharpey's fibres (which anchor the soft tissue to the bone), and ligament. The present study showed that PRP and BMSCs histologically promoted bone ingrowth into the tendon-bone interface. An incompletely mature chondral tendon-bone interface was observed in the BMSC + PRP group by H\&E and Russell-Movat pentachrome staining.

The walls of the bone tunnel in the PRP and BMSC + PRP groups showed more new bone formation than those of the control group. The newly formed bone represents a direct bonding area between the tendon and bone and prevents the knee instability associated with enlarging the bone tunnel. Enlargement of the bone tunnel at the articular end due to bone resorption is a common problem after ACL reconstruction [28]. New bone formation in the PRP and BMSC + PRP groups could be effective for long-term knee function by preventing the instability associated with bone tunnel enlargement.
The failure load in the BMSC + PRP group was significantly higher than that in the other two groups at 8 weeks postoperatively, but no difference was observed between the PRP and control groups. Tendon-bone healing after ACL reconstruction encompasses an orderly transition of necrosis of graft cells and ingrowth of host cells [13]. The cell types that initiate and regulate tendon-bone healing have not been positively identified until now [4]. Kobayashi et al. reported that if graft cells do not survive the first 2 weeks after ACL reconstruction, the graft will undergo necrosis [13]. It seems that the host cells from the surrounding bone marrow in the bone tunnel, which contains BMSCs and other preosteoblasts, contribute to tendon-bone interface repair $[13,14]$. In our study, significant differences in mechanical properties were observed between the BMSC + PRP and the other two groups, indicating that the seeded cells contribute to the tendon-bone healing process.

MSCs have become the gold standard for cellular therapies in musculoskeletal diseases because of their ease of expansion and capability of differentiating into chondrocytes, tenocytes, and osteocytes [29-31]. Several studies
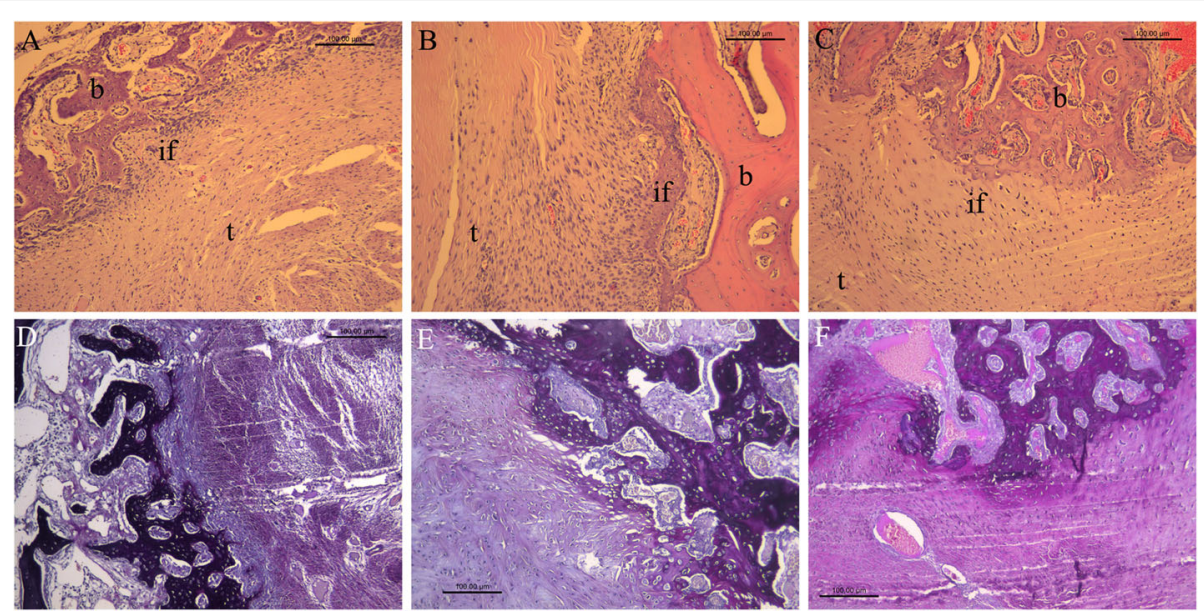

Fig. 6 Histological observations of the tendon-bone interface in the control $(\mathbf{a}, \mathbf{d})$, PRP $(\mathbf{b}, \mathbf{e})$, and BMSC + PRP groups $(\mathbf{c}$, $\mathbf{f})$ by haematoxylin and eosin (H\&E) (a-c) and Russell-Movat pentachrome staining (d-f). Magnification, $\times 100$; scale bar, $100 \mu \mathrm{m}$. $t$ tendon graft; $b$ bone; if interface 

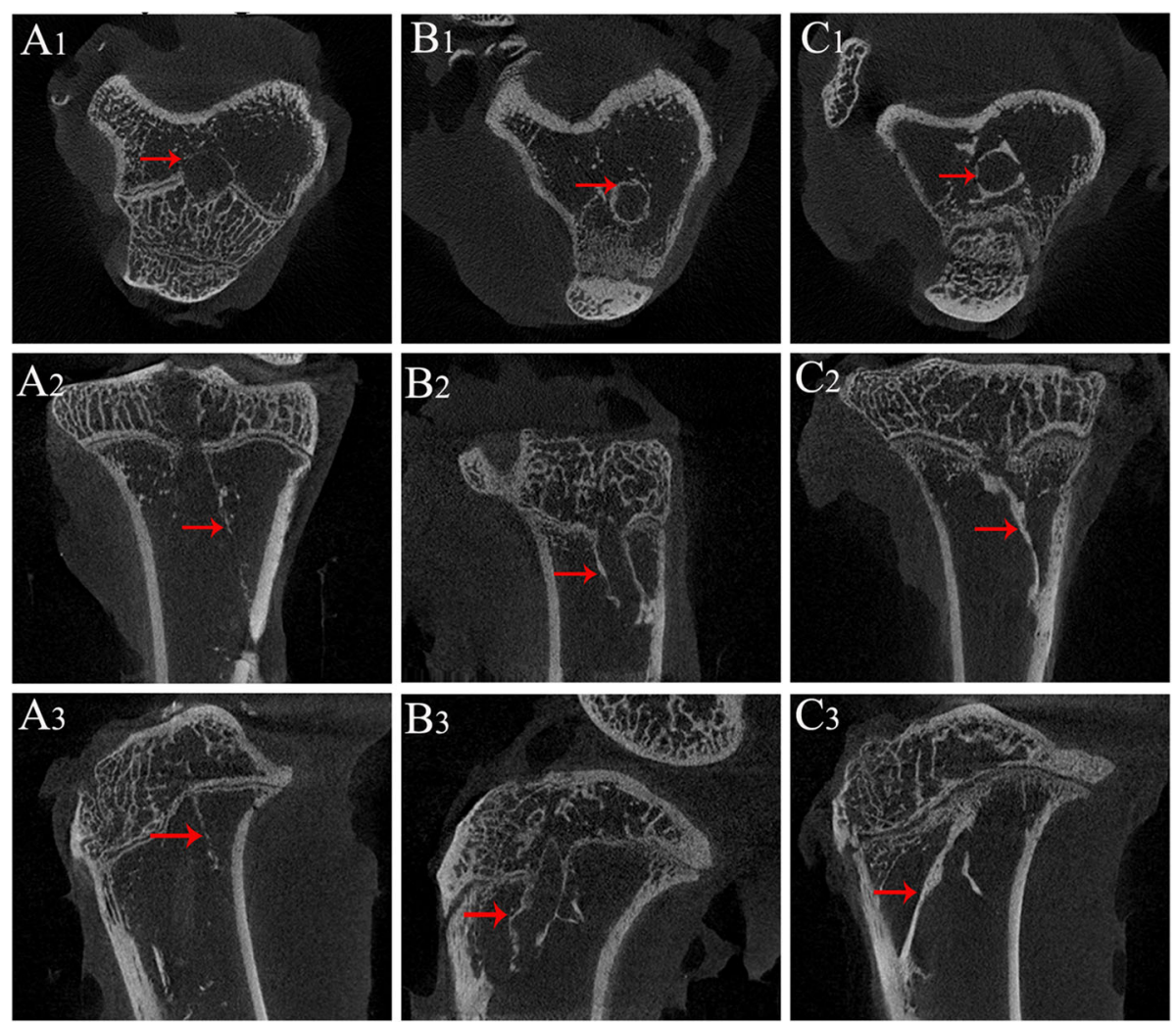

Fig. 7 Representative transverse (a1, b1, and $\mathbf{c 1}$ ), coronal (a2 , b2 , and $\mathbf{c 2}$ ), and sagittal (A3, b3 , and $\mathbf{c} 3$ ) section micro-computed tomography images in the three groups. No obvious mineralised tissue had formed in the tibial bone tunnels in the control group (a1-3). Clear mineralised tissue had formed at the tendon-bone interface in the PRP (b1-3) and BMSC + PRP groups (c1-3). The red arrows point to the newly formed mineralized tissue around the wall of the tibial bone tunnel

have explored the effects of tendon-bone healing with MSCs. Ouyang et al. applied BMSCs at the tendon-bone interface when fixing the hallucis longus tendon in a calcaneal bone tunnel. Only $50 \%$ of the tendon-bone interface contained fibrocartilage, although the BMSC group promoted collagen II staining [32]. Ju et al. explored the effect and mechanism of the implantation of BMSCs on tendon-bone healing in rats. The MSC group had a higher percentage of oblique fibres relative to the total interface area compared with controls [33]. Gulotta et al. reported that the addition of BMSCs to the tendon-bone interface did not improve the composition, structure, or strength of the tendon-bone attachment site [34]. It is possible that the tendon-bone interface lacks the molecular and/or cellular signals necessary for inducing the transplanted cells to appropriate differentiation, implying that MSC-based strategies should be combined with appropriate differentiation and
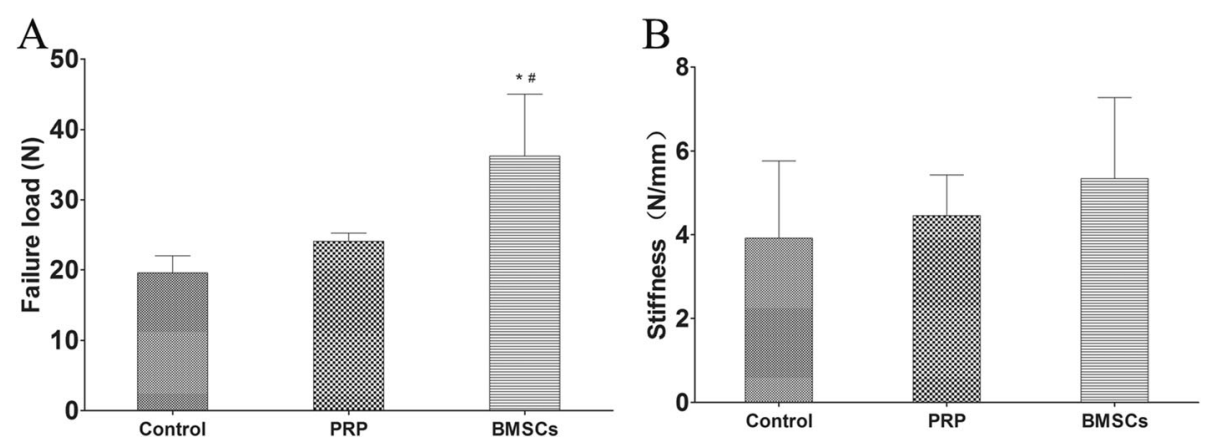

Fig. 8 Biomechanical test results: failure load (a) and stiffness (b). ${ }^{*} p<0.05$ vs. control group; ${ }^{*} p<0.05$ vs. the PRP group 
growth factors. In the present study, BMSCs serve as the material for regeneration, while PRP serves as the impetus to promote tendon-bone healing.

A limitation of this study was that the BMSCs were not labelled and tracked. Fan et al. labelled BMSCs with green fluorescence protein (GFP) to examine cell viability on scaffolds implanted into a rabbit knee joint for ACL reconstruction. Their results revealed GFP-positive scaffolds 4 weeks postoperatively, indicating that the BMSCs were viable [35]. Further studies are required to precisely track the fate of implanted BMSCs. Another limitation of this study was that the failure load was tested only under a static condition at $45^{\circ}$ of flexion. The biomechanical test was limited by our sample size and our Instron machine, which only tested tensile strength in a straight line.

\section{Conclusions}

PRP significantly stimulated osteogenic differentiation in BMSCs. The combination of PRP and BMSCs enhanced tendon-bone healing in a rabbit model of ACL reconstruction, demonstrating its potential for clinical use.

\section{Additional file}

Additional file 1: Results of biomechanical test. (PDF $94 \mathrm{~kb}$ )

\section{Acknowledgements}

This work was supported by the National Natural Science Foundation of China (81171687).

\section{Availability of data and materials}

All the data of the manuscript are presented in the paper or additional supporting files.

\section{Authors' contributions}

FB conceived and designed the experiment. $C T, C Z$, and DX performed the experiments. CT and FB analysed the data. CT contributed reagents/ materials/analysis tools. $\mathrm{CT}$ wrote the manuscript. All authors read and approved the final manuscript.

\section{Competing interests}

The authors declare that they have no competing interests.

\section{Consent for publication}

Not applicable.

\section{Ethics approval and consent to participate}

Animal experiments were approved by the Zhejiang University Ethics Committee.

\section{Author details}

${ }^{1}$ Department of Orthopaedic Surgery, The Fourth Affiliated Hospital, School of Medicine, Zhejiang University, Yiwu 322000, China. ${ }^{2}$ Department of Orthopaedic Surgery, The Second Affiliated Hospital, School of Medicine, Zhejiang University, Hangzhou 310009, China. ${ }^{3}$ Department of Orthopaedic Surgery, The First Affiliated Hospital of Zhengzhou University, Zhengzhou 450001, China.

Received: 19 June 2016 Accepted: 28 August 2016 Published online: 07 September 2016

\section{References}

1. Claes S, Verdonk P, Forsyth R, Bellemans J. The "ligamentization" process in anterior cruciate ligament reconstruction: what happens to the human graft? A systematic review of the literature. Am J Sports Med. 2011;39(11): 2476-83.

2. Fujita N, Kuroda R, Matsumoto T, Yamaguchi M, Yagi M, Matsumoto A, Kubo S, Matsushita T, Hoshino Y, Nishimoto K, et al. Comparison of the clinical outcome of double-bundle, anteromedial single-bundle, and posterolateral single-bundle anterior cruciate ligament reconstruction using hamstring tendon graft with minimum 2-year follow-up. Arthroscopy. 2011;27(7):906-13.

3. Tomita F, Yasuda K, Mikami S, Sakai T, Yamazaki S, Tohyama H. Comparisons of intraosseous graft healing between the doubled flexor tendon graft and the bone-patellar tendon-bone graft in anterior cruciate ligament reconstruction. Arthroscopy. 2001;17(5):461-76.

4. Bi F, Shi Z, Jiang S, Guo P, Yan S. Intermittently administered parathyroid hormone [1-34] promotes tendon-bone healing in a rat model. Int J Mol Sci. 2014;15(10):17366-79.

5. Eppley BL, Woodell JE, Higgins J. Platelet quantification and growth factor analysis from platelet-rich plasma: implications for wound healing. Plast Reconstr Surg. 2004;114(6):1502-8.

6. van den Dolder J, Mooren R, Vloon AP, Stoelinga PJ, Jansen JA. Platelet-rich plasma: quantification of growth factor levels and the effect on growth and differentiation of rat bone marrow cells. Tissue Eng. 2006;12(11):3067-73.

7. Kon E, Filardo G, Delcogliano M, Presti ML, Russo A, Bondi A, Di Martino A, Cenacchi A, Fornasari PM, Marcacci M. Platelet-rich plasma: new clinical application: a pilot study for treatment of jumper's knee. Injury. 2009;40(6): 598-603.

8. Hutchinson ID, Rodeo SA, Perrone GS, Murray MM. Can platelet-rich plasma enhance anterior cruciate ligament and meniscal repair? J Knee Surg. 2015; 28(1):19-28.

9. Chanda D, Kumar S, Ponnazhagan S. Therapeutic potential of adult bone marrow-derived mesenchymal stem cells in diseases of the skeleton. J Cell Biochem. 2010;111(2):249-57.

10. Chen X, Song XH, Yin Z, Zou XH, Wang LL, Hu H, Cao T, Zheng M, Ouyang HW. Stepwise differentiation of human embryonic stem cells promotes tendon regeneration by secreting fetal tendon matrix and differentiation factors. Stem Cells (Dayton, Ohio). 2009;27(6):1276-87.

11. Tucker BA, Karamsadkar SS, Khan WS, Pastides P. The role of bone marrow derived mesenchymal stem cells in sports injuries. J Stem Cells. 2010;5(4): 155-66.

12. Muruganandan S, Roman AA, Sinal CJ. Adipocyte differentiation of bone marrow-derived mesenchymal stem cells: cross talk with the osteoblastogenic program. Cell Mol Life Sci. 2009;66(2):236-53.

13. Kobayashi M, Watanabe N, Oshima Y, Kajikawa Y, Kawata M, Kubo T. The fate of host and graft cells in early healing of bone tunnel after tendon graft. Am J Sports Med. 2005;33(12):1892-7.

14. Kawamura S, Ying L, Kim HJ, Dynybil C, Rodeo SA. Macrophages accumulate in the early phase of tendon-bone healing. J Orthop Res. 2005;23(6):142532.

15. Zou J, Yuan C, Wu C, Cao C, Yang H. The effects of platelet-rich plasma on the osteogenic induction of bone marrow mesenchymal stem cells. Connect Tissue Res. 2014;55(4):304-9.

16. Atesok K, Fu FH, Wolf MR, Ochi M, Jazrawi LM, Doral MN, Lubowitz JH, Rodeo SA. Augmentation of tendon-to-bone healing. J Bone Joint Surg Am. 2014:96(6):513-21.

17. Wu W, Chen F, Liu Y, Ma Q, Mao T. Autologous injectable tissue-engineered cartilage by using platelet-rich plasma: experimental study in a rabbit model. J Oral Maxillofac Surg. 2007;65(10):1951-7.

18. Fan H, Hu Y, Zhang C, Li X, Lv R, Qin L, Zhu R. Cartilage regeneration using mesenchymal stem cells and a PLGA-gelatin/chondroitin/hyaluronate hybrid scaffold. Biomaterials. 2006;27(26):4573-80.

19. Yang L, Chang N, Liu X, Han Z, Zhu T, Li C, Yang L, Li L. Bone marrowderived mesenchymal stem cells differentiate to hepatic myofibroblasts by transforming growth factor-beta1 via sphingosine kinase/sphingosine 1phosphate (S1P)/S1P receptor axis. Am J Pathol. 2012;181(1):85-97.

20. Jenhani F, Durand V, Ben Azouna N, Thallet S, Ben Othmen T, Bejaoui M, Domenech J. Human cytokine expression profile in various conditioned media for in vitro expansion bone marrow and umbilical cord blood immunophenotyped mesenchymal stem cells. Transplant Proc. 2011;43(2):639-43.

21. Cheng K, Rai P, Plagov A, Lan X, Kumar D, Salhan D, Rehman S, Malhotra A, Bhargava K, Palestro CJ, et al. Transplantation of bone marrow-derived MSCs 
improves cisplatinum-induced renal injury through paracrine mechanisms. Exp Mol Pathol. 2013;94(3):466-73.

22. Anitua E, Andia I, Ardanza B, Nurden P, Nurden AT. Autologous platelets as a source of proteins for healing and tissue regeneration. Thromb Haemost. 2004;91(1):4-15.

23. de Mos $M$, van der Windt $A E$, Jahr $H$, van Schie $H T$, Weinans $H$, Verhaar JA, van Osch GJ. Can platelet-rich plasma enhance tendon repair? A cell culture study. Am J Sports Med. 2008;36(6):1171-8.

24. Rothrauff BB, Tuan RS. Cellular therapy in bone-tendon interface regeneration. Organogenesis. 2014;10(1):13-28.

25. Martinek V, Latterman C, Usas A, Abramowitch S, Woo SL, Fu FH, Huard J. Enhancement of tendon-bone integration of anterior cruciate ligament grafts with bone morphogenetic protein-2 gene transfer: a histological and biomechanical study. J Bone Joint Surg Am. 2002;84-a(7):1123-31.

26. Rodeo SA, Suzuki K, Deng XH, Wozney J, Warren RF. Use of recombinant human bone morphogenetic protein-2 to enhance tendon healing in a bone tunnel. Am J Sports Med. 1999;27(4):476-88.

27. Chen CH, Liu HW, Tsai CL, Yu CM, Lin IH, Hsiue GH. Photoencapsulation of bone morphogenetic protein-2 and periosteal progenitor cells improve tendon graft healing in a bone tunnel. Am J Sports Med. 2008;36(3):461-73.

28. Rodeo SA, Kawamura $S, M a C B$, Deng XH, Sussman PS, Hays P, Ying L. The effect of osteoclastic activity on tendon-to-bone healing: an experimental study in rabbits. J Bone Joint Surg Am. 2007:89(10):2250-9.

29. Awad HA, Butler DL, Boivin GP, Smith FN, Malaviya P, Huibregtse B, Caplan Al. Autologous mesenchymal stem cell-mediated repair of tendon. Tissue Eng. 1999;5(3):267-77.

30. Tuan RS, Boland G, Tuli R. Adult mesenchymal stem cells and cell-based tissue engineering. Arthritis Res Ther. 2003;5(1):32-45.

31. Kuo CK, Tuan RS. Mechanoactive tenogenic differentiation of human mesenchymal stem cells. Tissue Eng Part A. 2008;14(10):1615-27.

32. Ouyang HW, Goh JC, Lee EH. Use of bone marrow stromal cells for tendon graft-to-bone healing: histological and immunohistochemical studies in a rabbit model. Am J Sports Med. 2004;32(2):321-7.

33. Ju YJ, Muneta T, Yoshimura H, Koga H, Sekiya I. Synovial mesenchymal stem cells accelerate early remodeling of tendon-bone healing. Cell Tissue Res. 2008;332(3):469-78.

34. Gulotta LV, Kovacevic D, Ehteshami JR, Dagher E, Packer JD, Rodeo SA. Application of bone marrow-derived mesenchymal stem cells in a rotator cuff repair model. Am J Sports Med. 2009;37(11):2126-33.

35. Liu H, Fan H, Toh SL, Goh JC. A comparison of rabbit mesenchymal stem cells and anterior cruciate ligament fibroblasts responses on combined silk scaffolds. Biomaterials. 2008;29(10):1443-53.

\section{Submit your next manuscript to BioMed Central and we will help you at every step:}

- We accept pre-submission inquiries

- Our selector tool helps you to find the most relevant journal

- We provide round the clock customer support

- Convenient online submission

- Thorough peer review

- Inclusion in PubMed and all major indexing services

- Maximum visibility for your research

Submit your manuscript at www.biomedcentral.com/submit

) Biomed Central 